\title{
COMMENTS
}

\section{Comments on Rarity and Chaos, Thomas D. Rogers, Math. Biosci. 72:13-18 (1984)}

\author{
JOHN VANDERMEER \\ Division of Biological Sciences, \\ University of Michigan, \\ Ann Arbor, MI 48109
}

Rogers notes that 1 , a rare population need not be chaotic, and 2. a chaotic population need not be rare. He is, of course, correct, and if anyone had gotten some other impression from any of my work [1,2], I am grateful to Professor Rogers for clearing up that point.

On the other hand, I do hope his note does not confuse a second, possibly important, point with regard to chaotic behavior in population models. It has been noted that, for some models, a general correlation will exist between the size of population flushes and length of time the population remains rare after the flush, a condition I termed "resolved chaos" [2]. That result is unaffected by Rogers' comments, and whatever possible implications there are remain as previously discussed.

I also hope that the intended message of my perhaps incautiously titled "To be rare is to be chaotic" is not obscured. That message is simply that a possible (certainly neither necessary nor sufficient) cause of rarity is a chaotic population trajectory. Some models produce chaotic patterns in which population flushes are followed by long periods of rarity. This is offered as a third qualitative alternative to the usual interpretations of rarity - a low carrying capacity or a low equilibrium density as a result of interactions with other populations.

\section{REFERENCES}

$1 \mathrm{~J}$. Vandermeer, To be rare is to be chaotic. Ecology 63:1167-1168 (1982).

$2 \mathrm{~J}$. Vandermeer, On the resolution of chaos in population models. Theor. Popul. Biol. 22:17-27 (1982). 